\title{
Teaching and Learning Through the Use of Patient Cases Approach to the Psychiatric Patient: Case-Based Essays, Second Edition. By John W. Barnhill
}

\author{
American Psychiatric Association Publishing; Washington, DC; 2019; ISBN: 978-1-61537-002-3; \\ pp. 616; \$79 (paperback)
}

\author{
Mary K. Morreale ${ }^{1}$ (D)
}

Received: 12 February 2019 / Accepted: 19 February 2019 / Published online: 27 February 2019

(C) Academic Psychiatry 2019

"How can I make my teaching more engaging?" is a question many of us have likely asked ourselves as psychiatric educators. One tool to accomplish this task is to incorporate patient cases. Patient examples can add interest and applicability to didactic and small group activities and might even help learners recall information more readily.

Similarly, textbooks can be enhanced by the frequent use of patient cases. Approach to the Psychiatric Patient: Case-Based Essays, Second Edition is an excellent example. This comprehensive book revolves around ten diverse cases from which the editor and contributing authors explore a myriad of topics relevant to both students of psychiatry and psychiatrists themselves. Each case is followed by between 8 and 14 brief essays (all fewer than 1500 words), which are designed as "curbside consults" (p. xxxv). The material within this book is not meant to be read as the "final word" on any subject but as a starting point for thinking about the many approaches to patient care, "with a questioning attitude and pen in hand" (p. xxxvi).

The patient cases in the book span across age, psychiatric diagnosis, socioeconomic status, race, culture, and sexual identity. The specific topics covered are even broader in range and include straightforward subjects such as dialectical behavioral therapy, psychopharmacology of depression, and stimulant use, as well as more esoteric subjects like outsider art, the psychodynamics of psychosis, and an essay titled "Virginia Woolf, On Being Ill" (which compares the psychiatric illness, maternal loss,

Mary K. Morreale

mmorreale@med.wayne.edu

1 Wayne State University, Detroit, MI, USA and comfort in reading and writing presented in the patient case of an adolescent girl to the famous English writer, who died by suicide).

How might educators use Approach to the Psychiatric Patient: Case-Based Essays to enhance medical student and resident teaching? In a small group setting, a chapter (which contains one case and all applicable brief essays) could be discussed, with individuals assigned to specific, brief topics. As an example, following the case of an elderly man refusing an amputation, a discussion could ensue regarding capacity, the "psychiatric attitude," collaborative care, depression and medical illness, neurotrophins, biomarkers of depressive subtypes, interpersonal psychotherapy, service delivery, and geriatric depression. If all chapters were applied in this manner, a comprehensive picture of psychiatry would be easy to obtain. As a means to enhance didactics, the individual cases, which are written in a very detailed and descriptive manner, might be added.

Even if not used for teaching purposes, Approach to the Psychiatric Patient: Case-Based Essays is worth the read. It is well written and thought provoking, and it accomplishes the editor's objective to create a text that "converts large amounts of subspecialist complexity" into essays that are interesting and clear to digest.

\section{Compliance with Ethical Standards}

Disclosure The author states that there is no conflict of interest.

Publisher's Note Springer Nature remains neutral with regard to jurisdictional claims in published maps and institutional affiliations. 